\title{
Implicit Trust Propagation
}

\author{
Anuradha Yadav, Shampa Chakraverty, Ritu Sibal \\ \{yadavnsit, apmahs.nsit\}@gmail.com \\ Division of Computer Engineering, Netaji Subas Institute of Technology, \\ University of Delhi, India.
}

\begin{abstract}
The concept of trust plays key role in facilitating interactions and transactions in online systems. It is a major tool for handling information overload in online systems and filtering information by incorporating trust in recommender systems. Network of explicitly trusted users is very sparse. Implicit trust relations can be inferred based on user attributes and behavior. New trust relationships among users can be inferred using trust propagation properties which are not connected by explicit trust to solve the data sparsity problem.

Here, we evaluate how effective is indirect implicit trust in predicting the explicit trust using different metrics. We show that indirect implicit trust has relatively higher correlation with rating similarity compared to direct implicit trust. We are able to infer more trust relations using indirect implicit trust as seen from higher recall and coverage values but the accuracy in predicting trust is low compared to direct implicit trust.
\end{abstract}

Keywords: Trust properties, Implicit trust, Similarity trust, Explicit, Trust propagation.

\section{Introduction}

The rapid growth in quantity and diversity of information on the internet along with the explosive introduction of various services like buying products, blog feeds, auction, product comparison etc. has opened new avenues and offered new choices and services to users. This open nature of network has also led to problem of data overload which frequently overwhelm users, leads them to make bad decisions.

Recommender systems is being prominently used e-commerce. Quite a lot of techniques for recommendation have been proposed and Collaborative filtering $(\mathrm{CF})$ is being used frequently in recommendation algorithms. But application of CF has challenges associated with cold-start and sparsity of rating data to identify similar users.

Trust among users is an important piece of knowledge which can be utilized for solving the sparsity and cold start problem. The web of trust is too sparse to predict trust among unfamiliar 
users. Trust propagation is often used to infer trust between users with whom there was no interaction before and is claimed to be useful mean to solve data sparsity and cold start problem.

Researchers have proposed several approaches of using trust information. Main categorizations of trust are explicit vs. implicit trust and direct versus indirect trust. Explicit versus implicit trust and direct versus indirect trust are most commonly used categories of trust.

In [4],[1], trust relationships is being used to propose trust-aware recommender systems. They derive trust among users based on information from an online social network where users express how much they trust each other. This trust information helps to personalize the recommendations. Recommender systems use trust fromexplicit trust network. To improve the coverage, these papers use different trust propagation techniques.

Papagelis et al. [7] use the trust propagation on the implicit similarity trust to alleviate sparsity problem. They show recommendation quality using Mean Absolute Error (MEA) of predicted rating improve by incorporating the indirect implicit similarity trust in the recommender system. Their work was focused on the recommender system performance but did not study how indirect implicitly trusted actually compares with the explicit trust. This is key research gap we are addressing in this paper. In this work we study the trust propagation properties of implicit trust and assess the efficacy of indirect implicit trust in estimating the trust between users

We provide background on the relevant trust types and recommender systems in section 2 . In sections 3 we define a methodology and data used for our study. Then in section 4 we provide the results. Finally, we conclude our result and explore future research directions in Section.5.

\section{Prior Work}

Throghout the paper we use the following representation. $I, U$ and $R$ represent set of items, users and ratings, respectively. We use symbols $i, j$ for items, $u, v$ for users. $r_{u, i}$ denotes a rating given by user $u$ on item $i$. Let $I_{u}$ be the set of items rated by user $u$, and $t_{u, v}$ be the trustworthiness of user $u$ towards user $v$ and $s_{u, v}$ be the rating similarity between user $v$ and $u$.

As there are multiple types of trust defined in the research literature, it is important to define the trust types that are focus of this paper. We focus on two types of trust metrics, direct and indirect implicit similarity trust. 
Trust can be categorized as explicit or implicit based on how it is expressed [10][9]. Trust can be called explicit where trust is expressed explicitly by the user. Users assign subjective trust statement to other users based on their past experiences, behaviors and preferences and attitudes. This trust statement can have different values like categorical, binary, multinomial. Explicit trust is user's self-expressed statement of trust in another user

Whereas implicit trust metrics estimate the trust among two members based on their profile attributes, behavior, attitudes and direct interaction between them. Implicit trust is not the user's self-expressed statement of trust but a metric calculated by an external party. Implicit trust metrics provide much more richer information about the trust relations in the online social network than explicit trust [9].

As in the real world, online social networks trust is also based on either own experience or on the recommendations provided by other users or combination of both. This leads to classification of trust in direct and indirect trust.

Direct trust is derived from the direct experience of the member with the other users. Direct trust is expressed explicitly by the trustor or is computed implicitly on the basis of experiences, behavior and attitudes of the trustee.

Indirect trust is derived on the basis of experience of other members in the social network with the third party. Indirect trust is computed using propagation along network path. Examples of indirect trust are tidal trust[4] and mole trust[1].

Combination of these different trust classifications can be used to define direct explicit trust or direct implicit trust. Further if trust is implicitly derived from similarity in some user attribute we classify trust as direct implicit trust or indirect implicit similarity trust.

Papagelis et al. [7] use this concept of indirect implicit similarity trust. They proposed a method for alleviating data sparsity problem in CF using inferring implicit trust among users derived from rating similarity and then exploiting the trust propagation techniques to extend this trust inference to even users which do not have direct connection.

Papagelis et al.[7] applied the Pearson Correlation Coefficient (PCC) to predict trust among users. They propose that rating correlation can be used as measure of trust in place of similarity, and therefore theory of trust propagation can be used to predict trust among users who have no co- 
rated items. Their approach is a solution to the data sparsity problem in conventional similarity based CF systems.

$$
t_{u, v}=s_{u, v}=\frac{\sum_{i}\left(r_{u, i}-\bar{r}_{u}\right)\left(r_{v, i}-\bar{r}_{v}\right)}{\sqrt{\sum_{i}\left(r_{u, i}-\bar{r}_{u}\right)^{2}} \sqrt{\sum_{i}\left(r_{v, i}-\bar{r}_{v}\right)^{2}}}
$$

Where $s_{u, v}$ is the rating similarity among user $u$ and $v$, which also indicated trust $t_{u, v}$. For trust propagation they proposed the method given by equation (2). If $u$ trusts $v$ and $v$ trusts $w$ then trust value between $u$ and $w$ is,

$$
t_{u, w}=t_{u, v} \oplus t_{v, w}=\frac{\# I_{u, v} t_{u, v}+\# I_{v, w} t_{v, w}}{\# I_{u, v}+\# I_{v, w}}
$$

In addition to the PCC similarity based trust we have also used the mean absolute difference (MAD) based trust metric for our analysis and used that same propagation technique to both the implicit trusts. Chakraverty et al. [3] use Mean of Absolute (MAD) similarity among two members $u$ and $v$ is defined by the following equation:

$$
t_{u, v}=\frac{1}{\# I_{u, v}} \sum_{i \in I_{u, v}}\left|r_{u, i}-r_{v, i}\right|
$$

As trust inferences are constructed on traversal paths in a network, it is possible to find multiple paths that connect two customers. Papagelis et al. [7] define multiple strategies for computing indirect trust between users when multiple traversal paths exist amid the source and target user. For simplistic we have used trust path composition using average of all the paths for our study.

\section{Methodology}

We extend the analysis of Chakraverty et al.[3] where they study the direct implicit similarity trust to the indirect implicit similarity trust used by Papagelis et al.[7]. We perform analysis of indirect implicit similarity trust. To find the relationship among rating similarity and trust we use Epinion dataset and for effectiveness of predicting trust among members through propagation of implicit trust inferred from rating similarity. Details of the dataset are given in [3]. The Epinion dataset contains binary information related to explicit trust between users in form of trust and no-trust. We find binary implicit trust among members, to evaluate how effective is rating similarity in inferring trust. Direct and indirect implicit trust computed by equation 1, 2and 3 is continuous 
valued. Threshold-based method, as given in equation (4) proposed by Yuan et al. [11] is used to have binary values

$$
t_{u, v}=\left\{\begin{array}{cc}
1, & s_{u, v}>\tau^{\operatorname{sim}} \& \& \# I_{u, v}>\tau^{I c o u n t} \\
0, & \text { otherwise }
\end{array}\right.
$$

Where:

- $\quad s_{u, v}$ is rating similarity among memebers $u, v . s_{u, v}$ may represent either PCC or MAD.

- $\tau^{\text {sim }}$ parameter is used for preset threshold. For establishing positive trust among members, this decides the level of similarity. $\tau^{\text {sim }}$ can be used as $\tau^{\mathrm{MAD}}$ or $\tau^{\mathrm{PCC}}$. Thus, two user's $\mathrm{u}, \mathrm{v}$ trusts each other, if PCC similarity value is above the preset threshold $\tau^{\mathrm{PCC}}$ or MAD similarity value is below the preset threshold $\tau^{\mathrm{MAD}}$.

- $\tau^{\text {Icount }}$ parameter is the minimum value that must be co-rated users .

To validate how effective is indirect implicit similarity against explicit trust, we compare both the derived implicit trust and the explicit trust available in the dataset. We use $P_{u}$ for set of trusted predicted users for a given user $u$. For a given user $\mathrm{u} T_{u}$ represents the all explicit trusted users. Let $Z=\left\{u \in U \mid P_{u} \neq \emptyset\right\}$ is a set of those users for which at least one user is correctly predicted using rating similarity. To evaluate our prediction results following metrics are used:

$$
\begin{gathered}
\text { coverage }=\frac{\# Z}{\# U} \\
\text { precision }=\frac{1}{\# Z} \sum_{u \in Z} \frac{\#\left(P_{u} \cap T_{u}\right)}{\# P_{u}} \\
\text { recall }=\frac{1}{\# Z} \sum_{u \in Z} \frac{\#\left(P_{u} \cap T_{u}\right)}{\# T_{u}}
\end{gathered}
$$

\section{Results}

We used rating similarity to predict implicitly trusted users for both direct and indirect trust using equation (4). Coverage, precision and recall metrics are being used to evaluate the performance of prediction for direct and indirect implicit trusted users against explicit trusted users. Different values of similarity threshold $\left(\tau^{\mathrm{PCC}}, \tau^{\mathrm{MAD}}\right)$ and co rated item count threshold 
$\left(\tau^{\text {Icount }}\right)$ used to predict effect of rating similarity on prediction accuracy. Coverage, precision and recall for indirect implicit trust generated after trust propagation with the baseline case of direct implicit trust from Chakraverty et al.[3]. Figure 1 and Table 1 depicts the baseline value for coverage precision and recall of direct implicit trust from Chakraverty et al. [3].

Table 1. Precision, Coverage and Recall results for direct implicit similarity trust

\begin{tabular}{|c|c|c|c|c|c|c|c|}
\hline $\begin{array}{c}\text { Indep } \\
\text { Variable }\end{array}$ & $\begin{array}{c}\text { Control } \\
\text { Variable } \\
\end{array}$ & \multicolumn{2}{|c|}{ Precision } & \multicolumn{2}{|c|}{ Recall } & \multicolumn{2}{|c|}{ Coverage } \\
\hline$\tau^{\text {sim }}$ & $\tau^{\text {Icount }}$ & $\begin{array}{c}\text { Initial, } \\
\text { Final } \\
(\%)\end{array}$ & $\begin{array}{c}\text { \%age } \\
\text { Variat } \\
\text { ion }\end{array}$ & $\begin{array}{c}\text { Initial, } \\
\text { Final } \\
(\%)\end{array}$ & $\begin{array}{c}\text { \%age } \\
\text { Variat } \\
\text { ion }\end{array}$ & $\begin{array}{c}\text { Initial, } \\
\text { Final } \\
(\%)\end{array}$ & $\begin{array}{c}\text { \%age } \\
\text { Variat } \\
\text { ion }\end{array}$ \\
\hline \multirow{3}{*}{$\tau^{\mathrm{PCC}}$} & 2 & $3.2,3.1$ & -1.3 & $5.6,1.9$ & -67.0 & $19.6,16.6$ & -15.5 \\
\hline & 5 & $10.2,10.3$ & 0.7 & $5.2,0.9$ & -83.2 & $5.8,3.6$ & -37.5 \\
\hline & 10 & $21,23.3$ & 11.0 & $5.5,0.7$ & -87.6 & $1.7,0.6$ & -66.1 \\
\hline \multirow{3}{*}{$\tau^{\mathrm{MAD}}$} & 2 & $3.2,3.8$ & 17.9 & $5.7,1.1$ & -79.9 & $19.7,14.4$ & -27.1 \\
\hline & 5 & $10.3,12.8$ & 24.9 & $0.9,5.2$ & -83.5 & $3,5.8$ & -48.4 \\
\hline & 10 & $21,23.8$ & 13.3 & $0.7,5.5$ & -86.6 & $0.6,1.7$ & -65.9 \\
\hline
\end{tabular}

(a) Precision
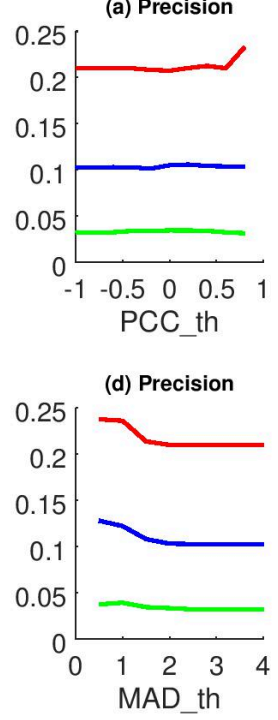

(b) Recall

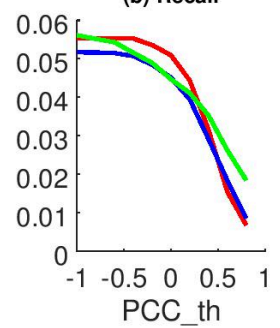

(e) Recall

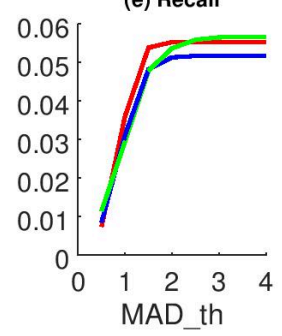

(c) Coverage
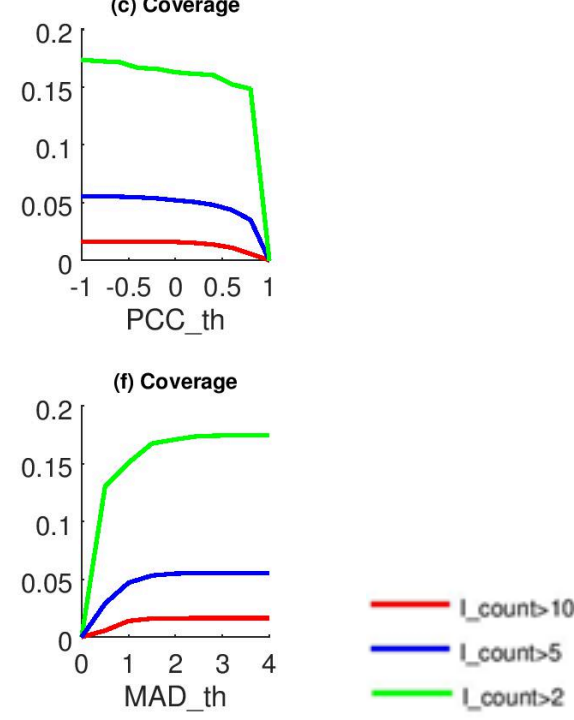

Figure 1. Precision, Recall and Coverage vs Similarity for direct implicit similarity trust 
(a) Precision

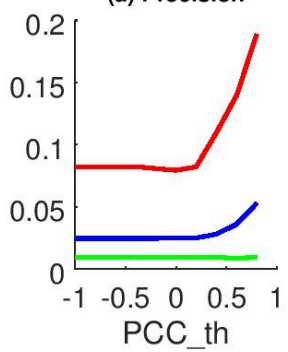

(d) Precision

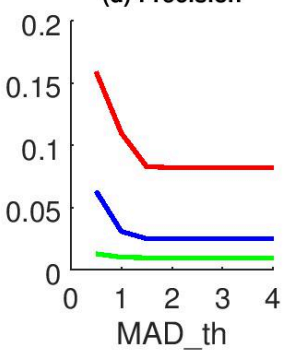

(b) Recall

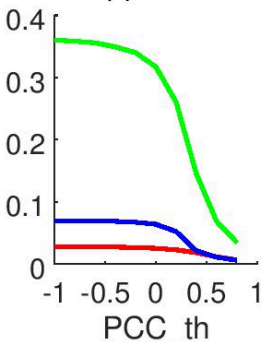

(e) Recall

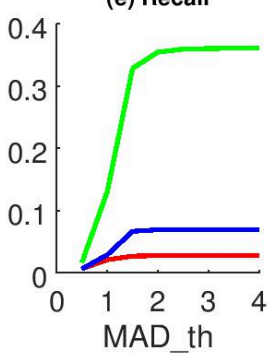

(c) Coverage

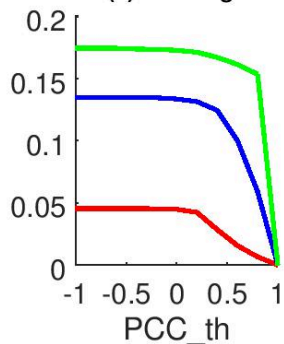

(f) Coverage

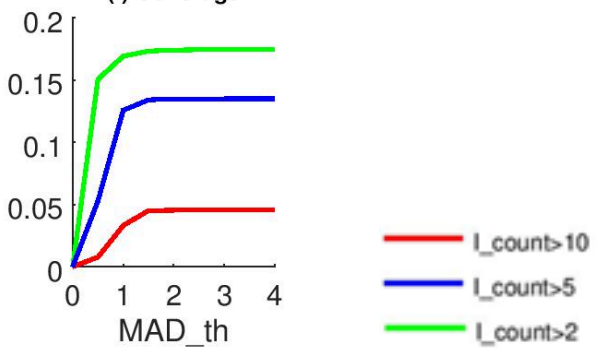

Figure 2. Precision, Recall \& Coverage vs Similarity for indirect implicit similarity trust

Table 2. Precision, Coverage and Recall results for indirect implicit similarity trust

\begin{tabular}{|c|c|c|c|c|c|c|c|}
\hline $\begin{array}{c}\text { Indep } \\
\text { Variable }\end{array}$ & $\begin{array}{c}\text { Control } \\
\text { Variable }\end{array}$ & \multicolumn{2}{|c|}{ Precision } & \multicolumn{2}{c|}{ Recall } & \multicolumn{2}{c|}{ Coverage } \\
\hline \multirow{4}{*}{$\tau^{\text {sim }}$} & \multirow{2}{*}{$\begin{array}{c}\text { Initial, } \\
\text { Icount }\end{array}$} & $\begin{array}{c}\text { Final } \\
(\mathbf{\%})\end{array}$ & $\begin{array}{c}\text { \%age } \\
\text { Variat } \\
\text { ion }\end{array}$ & $\begin{array}{c}\text { Initial, } \\
\text { Final } \\
(\%)\end{array}$ & $\begin{array}{c}\text { \%age } \\
\text { Variat } \\
\text { ion }\end{array}$ & $\begin{array}{c}\text { Initial, } \\
\text { Final } \\
(\%)\end{array}$ & $\begin{array}{c}\text { \%age } \\
\text { Variat } \\
\text { ion }\end{array}$ \\
\hline \multirow{4}{*}{$\tau^{\text {PCC }}$} & 2 & $0.9,0.9$ & -9.8 & $36,3.5$ & -90.3 & $17.4,15.3$ & -12 \\
\cline { 2 - 8 } & $\mathbf{5}$ & $\mathbf{2 . 5 , 5 . 3}$ & $\mathbf{1 1 5 . 3}$ & $\mathbf{6 . 9 , 0 . 6}$ & $\mathbf{- 9 1 . 2}$ & $\mathbf{1 3 . 5 , 5 . 9}$ & $\mathbf{- 5 6 . 3}$ \\
\cline { 2 - 8 } & 10 & $8.2,18.9$ & 129.5 & $2.7,0.7$ & -75.7 & $4.5,0.7$ & -85.6 \\
\hline \multirow{3}{*}{$\tau^{\text {MAD }}$} & 2 & $1.0,1.3$ & 33.9 & $36.1,1.7$ & -95.4 & $17.5,15.1$ & -13.7 \\
\cline { 2 - 8 } & $\mathbf{5}$ & $\mathbf{2 . 5 , 6 . 3}$ & $\mathbf{1 5 5 . 8}$ & $\mathbf{6 . 9 , 0 . 6}$ & $\mathbf{- 9 1 . 3}$ & $\mathbf{1 3 . 3 , 5 . 3}$ & $\mathbf{- 6 0 . 8}$ \\
\cline { 2 - 8 } & 10 & $8.2,15.9$ & 93.2 & $2.7,0.6$ & -76.4 & $4.5,0.8$ & -83.2 \\
\hline
\end{tabular}

Figure 2 and Table 2 depicts the value of coverage precision and recall for indirect implicit trust. Precision, recall and coverage are shown in Figures $2 \mathrm{a}, 2 \mathrm{~b}$, and $2 \mathrm{c}$ for different values of $\tau^{\text {PCC }}$ corresponding to three fixed values of $\tau^{\text {Icount }}$ viz., 2,5 and 10 . Figures $2 \mathrm{~d}, 2 \mathrm{e}$, and $2 \mathrm{f}$ show the values of coverage precision, recall for varying $\tau^{\mathrm{MAD}}$ for three different fixed values of $\tau^{\mathrm{Icount}}$ viz., 2,5 and 10. Table 2 shows the values of precision, recall and coverage, for the min value of 
$\tau^{\mathrm{PCC}}(-1)$ and the max value of $\tau^{\mathrm{PCC}}(0.8)$, and the consequent percentage variation over this range. Also, we record the values of coverage, precision, recall for the min value of $\tau^{\mathrm{MAD}}$ (4) and the $\max$ value of $\tau^{\mathrm{MAD}}(0.5)$.

We observed that irrespective of $\tau^{\text {Icount }}$ precision, recall and coverage does not change. We set $\tau^{\text {Icount }}=5$ for observations:

- $\tau^{\text {PCC }}$ and $\tau^{\mathrm{MAD}}$ throughout the range remains very low. For $\tau^{\mathrm{PCC}}$ highest value of precision and coverage is $5.3 \%$ and $13.5 \%$ respectively. The highest value of precision and coverage is $6.3 \%$ and $13.3 \%$ respectively for $\tau^{\mathrm{MAD}}$. Maximum value of recall is $6.9 \%$ for both $\tau^{\mathrm{MAD}}$ and $\tau^{\mathrm{PCC}}$.

- Recall has improved for indirect implicit trust as compared to direct implicit trust for its maximum value and does not improve even for minimum preset value of similarity threshold $(\mathrm{PCC}=-1)$. Reason being is as many user pairs do not have many co-rated items even after trust propagation:

- Premise of implicit trust is number of co-rated items among two users. PCC and MAD are calculated from ratings given to a common set of items by users. Though, even after propagation, users explicit trust network fails for similarity trust prediction but rated different number of items.

- We have precluded similarity calculated on 2 common items as it is not reliable. Above observations shows that, the trust among some users cannot be calculated even when the similarity threshold is at minimum level.

- There is significant improvement in precision as similarity threshold $\tau^{\mathrm{PCC}}$ is increased $\tau^{\mathrm{MAD}}$ is decreased, indicating a strong correlation with similarity. Precision improves by $115.3 \%$ and $155.8 \%$ for $\tau^{\mathrm{PCC}}$ and $\tau^{\mathrm{MAD}}$ respectively. Though this does not mean that indirect implicit trust can predict trust more precisely than direct trust for higher similarity values. Infact indirect implicit trust is relative less precise in predicting trust compared to direct similarity trust as low similarity values

- In the case of both $\tau^{\mathrm{PCC}}$ and $\tau^{\mathrm{MAD}}$ increase in the value of similarity threshold leads to decrease in maximum recall $6.9 \%$. 
- Maximum coverage value of $13.5 \%$ and $13.3 \%$ for $\tau^{\mathrm{PCC}}$ and $\tau^{\mathrm{MAD}}$ respectively drops gradually as similarity threshold increased.

From above observations, we derive that:

- Indirect implicit trust inferred by propagating implicit trust can help in improving significantly improving the coverage of trust user pairs.

- Low value of precision indicates that propagation of implicit similarity trust is not as effective in predicting trust as direct implicit trust.

\section{Discussion \& Conclusion}

We derive that indirect implicit trust can be useful in inferring trust between users when other direct or explicit measures of trust are not available. The precision of indirect implicit trust derived from rating similarity is highly correlated with the level of similarity among users. But in real world data set the absolute value of precision is low. Indirect implicit trust can be useful in applications like recommender systems for solving data sparsity and cold start problems. We believe results of inferring indirect implicit trust among memebers based on the one specific propagation technique that we studied are very promising and in future different propagation method can be tried. In future in addition to the similarity trust other types of implicit trust like relational implicit trust metrics can be used with trust propagation.

\section{References}

[1] Avesani, P., Massa, P., Tiella, R.: A Trust enhanced Recommender System application: Moleskiing, In Proceedings of ACM SAC, p 1589-1593. (2005)

[2] Bobadilla, J., Ortega F., Hernando, A., Gutiérrez, A.: Recommender systems survey, Knowledge Based Systems, 46, 109-132. (2013)

[3] Chakraverty, S., Yadav, A., Sibal, R.: On evaluating the effectiveness of rating similarity-based trust, Social Netw. Analys. Mining 6(1), 98:1-98:13, (2016)

[4] Golbeck, J.A.: Computing and applying trust in web-based social networks, Ph.D. Dissertation, University of Maryland, College Park, ISBN:0-542-18324-2 (2005)

[5] Jøsang, A., Ismail, R., Boyd, C.: A survey of trust and reputation systems for online service provision, Decision Support Systems 43 (2), 618-644 (2007) 
[6] Liu, H., Lim, E. P., Lauw, H. W., Le, M. T., Sun, A., Srivastava, J., Kim, Y. A.: Predicting trusts among users of online communities: An epinions case study, In Proceedings of the 9th ACM Conference on Electronic Commerce (EC), ACM Press, New York, p 310-319, (2008)

[7] Papagelis, M., Plexousakis, D., Kutsuras, T.: Alleviating the Sparsity Problem of Collaborative Filtering Using Trust Inferences, iTrust, p 224-239, (2005)

[8] Tang, J., Gao, H., Liu, H.: mtrust: discerning multi-faceted trust in a connected world. In Proceedings of the fifth ACM international conference on Web search and data mining, pages 93102. ACM, (2012)

[9] Guo, G., Zhang, J., Thalmann, D., Basu, A., and Yorke-Smith, N.: From Ratings to Trust: an Empirical Study of Implicit Trust in Recommender Systems, Symposium on Applied Computing Recommender Systems, (2014)

[10] Tavakolifard, M.: On Some Challenges for Online Trust and Reputation Systems, PHD Thesis, Norwegian University of Science and Technology, (2012)

[11] Yuan, W., Shu, L., Chao, H. C., Guan, D., Lee, Y. K., Lee, S.: ITARS: trust-aware recommender system using implicit trust networks, IET Communications, 4(14), 1709-1721, (2010) 